\title{
Surgical Management of Tempromandibular Joint Ankylosis: An analysis of 20 cases
}

\author{
Bhatnagar $\mathbf{S}^{1}$, Navaneetham $\mathrm{A}^{2}$ \\ ${ }^{1}$ Dr Shweta Bhatnagar, Assistant Professor, Department of Dentistry, Bundelkhand Medical College Sagar,Madhya Pradesh, \\ India. ${ }^{2}$ Dr Anuradha Navneetham, Professor, B R Ambedkar Dental College, Bangalore, Karnatka.
}

Address for correspondance: Dr. Shweta Bhatnagar, Email: shwetakeshav@gmail.com

\begin{abstract}
Introduction: Ankylosis is a Greek term meaning "stiff joint". The fusion of the articular surfaces of head of condyle with the glenoid fossa leads to TMJ ankylosis. The subarticular layer is composed of a rich vascular network that penetrates the cortical layer. Haemarthrosis of this highly osteogenic layer due to trauma commonly leads to the organization of fibroosseous mass and bony ankylosis can ensue. Method: This clinical study included 22 patients from 2008-2013. Out of these 20 patients underwent surgery. There were 17 patients below 14 years of age and five patients above 14 years. Etiopathogenesis was traumatic in 19 cases, septic in 2 cases and autoimmune in one case. Results: The patients were followed up for one to six years. All patients under treatment showed a distinctive improvement both in articular functionality and symptoms. In two patients recurrence was noticed and they were taken for secondary surgery. The functional results of using temporalis fascia as an interpositional material were satisfactory. Conclusion: We conclude that early postoperative physiotherapy and strict follow-up play an important role in preventing postoperative adhesions
\end{abstract}

Key words: TMJ Ankylosis, Interpositional Arthroplasty, Andegump Deformity

\section{Introduction}

Ankylosis is a Greek term meaning "stiff joint". The fusion of the articular surfaces of head of condyle with the glenoid fossa leads to TMJ ankylosis. The ankylosis may be bony or fibrous depending on the tissue bridging the articular surfaces. It may be a sequel of trauma, infection, autoimmune diseases. The subarticular layer is composed of a rich vascular network that penetrates the cortical layer. Haemarthrosis of this highly osteogenic layer due to trauma commonly leads to the organization of fibroosseous mass and bony ankylosis can ensue [1]. The purpose of this study is to analyze and to evaluate the efficacy of temporalis fascia as an interpositional graft material.

\section{Methods}

This retrospective clinical study included 22 patients which were diagnosed with TMJ Ankylosis. Out of these 20 patients underwent Interpositional Arthroplasty with temporalis fascia from 2008-2013. Patients were followed up for one to six years. Out of 22 patients 17 were below 14 years $(11 \mathrm{M}, 6 \mathrm{~F})$ and 5 were above 14 years $(4 \mathrm{M}, 1 \mathrm{~F})$. There were 13 cases of unilateral and 9 cases of bilateral TMJ

Manuscript received: $16^{\text {th }}$ Oct 2013

Reviewed: $29^{\text {th }}$ Nov 2013

Author Corrected: $13^{\text {th }}$ Dec 2013

Accepted for Publication: $11^{\text {th }}$ Feb 2014
Ankylosis. Etiopathogenesis was traumatic in 19 cases ( Among these parents of 10 patients reported delivery was done at home and inability to open mouth was noticed few months after birth suggesting birth trauma as a probable cause ), Septic in 2 cases and autoimmune in one case. Among 9 cases of bilateral TMJ ankylosis 3 patients had repetitive nocturnal airway obstruction leading to increased day time somnolence, fatigue and a severe obstructive sleep apnea syndrome.

The protocol for surgery was as follow

1. Aggressive excision of ankylotic mass.

2. Coronoidectomy on affected side.

3. Coronoidectomy on contralateral side if mouth opening was less than $35 \mathrm{~mm}$ or to the point of dislocation of unaffected TMJ.

4. Lining of Tempromandibular joint with temporalis fascia.

5. Reconstruction of ramus condylar unit with costochondral graft and rigid fixation in children.

6. Early mobilization of jaw

7. Excessive physiotherapy 
Research Article

A modified Alkayat and Bramley Approach was used in which incision was made inside the tragus to hide the incision in the external ear with the temporal extension was used.

The dissection was done up to the temproparietal fascia. Above the zygomatic arch the superficial layer of temporalis fascia was incised in an oblique line running from the tragus to superior temporal line. A mosquito forcep was used to dissect along the anteromedial direction to the TMJ region. At this point Sharp dissection is done along the root of zygoma to the periosteum to reach the ankylotic mass.

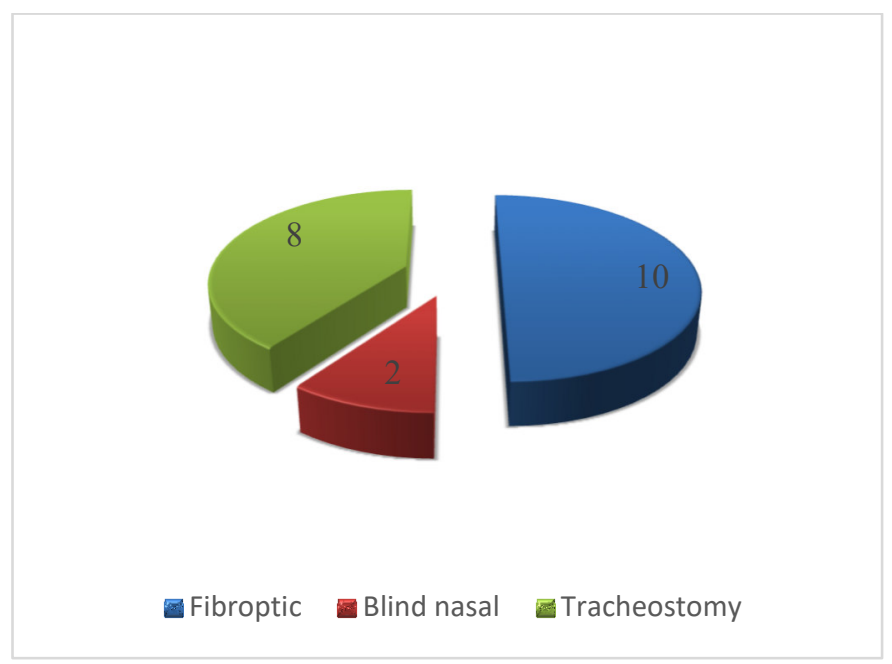

Fig 1: Modes of intubation

\section{Results}

Nineteen patients maintained a stable mouth opening in range of $30 \mathrm{~mm}$ to $40 \mathrm{~mm}$ postoperatively. One child patient mouth opening reduced to $15 \mathrm{~mm}$ and fibrous ankylosis ensued because of non compatibility with post operative physiotherapy.

The release of ankylosis greatly relived the sleep apnea and all three patients reported complete relief from day time sleepiness and fatigue. One of these patients developed post extubation pulmonary edema and required ventilatery support for 48 hours post operatively. The distraction osteogenesis is planned in these cases.

\begin{tabular}{|l|l|}
\hline Outcome & No. of patients \\
\hline $\begin{array}{l}\text { Good mouth opening (more } \\
\text { than } 30 \mathrm{~mm} \text { ) }\end{array}$ & 19 \\
\hline $\begin{array}{l}\text { Poor mouth opening (less } \\
\text { than } 20 \mathrm{~mm} \text { ) }\end{array}$ & 1 \\
\hline No follow up & 2 \\
\hline
\end{tabular}

\section{Discussion}

The majority of patients with TMJ Ankylosis are from rural areas where the patient awareness is less. Most of the parents were ignorant of birth trauma during delivery done at home or injury to the TMJ due to fall and violence. The condition is usually discovered accidently by parents when they observe the child is unable to eat. L Valentini et al noticed that the etiopathogenesis of TMJ ankylosis was traumatic in $83 \%$ cases, autoimmune in $9 \%$ and septic in $9 \%$ cases [2]. In our study we found the etiopathogenesis was traumatic in $86 \%$ cases, autoimmune in $5 \%$, Septic in $9 \%$ cases. TMJ ankylosis start early in life, leads to the serious esthetic deformities and functional problems.

The unilateral ankylosis leads to the unilateral hypoplastic mandible with chin deviating towards the affected side. In case of long standing ankylosis maxillary growth may be hampered due to lack of functional matrix stimulation for maxilla leading to the occlusal canting. Oral hygiene is difficult to maintain. Eruption of permanent teeth may also be impeded because of lack of space. The bilateral TMJ Ankylosis will lead to bird fascies or andegump deformity. A small retrognathic mandible will be present as contained arch.

The small retrognathic mandible along with the short rami and decreased bigonial distance leads to a very narrow airway which when compounded with tongue fall during sleep leads to a severe obstructive sleep apnea syndrome. The patient will give a history of snoring, excessive day time sleepiness and fatigue. The long standing ankylosis also results in isometric contractions of muscle of mastication. This leads to elongation and thickening of coronoid process under effect of temporalis Muscle, shortening of Mandibular ramus (pterygomassetric sling), development of prominent antegonial notch owing to antagonistic action of pterygomassetric sling and depressor muscles. Recession of chin will occur under effect of suprahyoid depressor Muscles.

The excessively retrognathic mandible along with tongue fall leads to the obstructive sleep apnea syndrome [3]. In our study 3 patients had severe airway compromise. One of the patients could not sleep in supine position and has to sleep at prone position throughout night.

Digital palpation of the ankylosed temporomandibular joint during maximal movements demonstrates none or very limited translation of the condyle[4-6]. Gap arthroplasty and interpositional arthroplasty are suggested as the treatment modalities. Topazian reviewed gap arthroplasty without interposition and reported a recurrence rate of 53\% [7]. 
Research Article

Most authors tend to agree, however, that recurrent ankylosis is less likely if material is interposed between the divided bone ends. For interpositional arthroplasty, broadly two options are available and popular following resection of ankylosed segment: prosthetic implants and autogenous grafts. Proplast-Teflon TMJ implants were popular until 1990. After 1990, it has been established that implants cause a foreign body giant cell reaction, and the US Food and Drug Administration issued a nation-wide safety alert [810]. We used temporalis fascia an autograft as an interpositional material.

The advantages of using temporalis fascia are that it is available in same surgical site, middle temporal artery, a constant branch of superficial temporal artery. Temporalis fascia flap is a locally available axial pattern flap, easy to elevate and available in all clinical situations, and being an autograft there are less chances of rejection. Hassan et al concluded that deep temporal fascia is vascularized flap has fewer chances of subsequent absorption and fibrosis [11, $12]$.

One of our patients had autoimmune Marie strumpell disease. The rate of TMJ ankylosis in Marie Strumpell Disease is not exactly known. The incidence of marie strumpell disease is $1 \%$. The patient presented with no mouth opening, no neck movements, low back pain and stiffness, poor chest expansion and exaggerated dorsal kyphosis. The involvement of sacroiliac joint is hallmark of disease.

The patient classically had a bamboo spine appearance on lateral spine x-ray [13]. The orientation of surgical plane was difficult to achieve as neck was fused in flexion, not allowing the patient positioning. The tissue planes were difficult to separate probably because of fibrosis involving the various layers.

Irrespective of etiology all patients responded well to the protocol and are maintaining good mouth opening and function.

The temporalis fascia is autogenous, resilient, vascularized flap in the proximity to the joint, which allows a pedicle transfer of vascularized tissue into the joint area and makes it a viable alternative [14].

\section{Conclusion}

We conclude that temporalis fascia is a viable interpositional material which is available in the surgical field and give good results when used as an interpositional material.

\section{Funding: Nil \\ Conflict of interest: Nil \\ Permission from IRB: Yes}

\section{References}

1. Orhan Guven. A clinical study on temporomandibular joint ankylosis in children. "The journal of Craniofacial Surgery.2008;19(5):1263-9

2. Valentini V, Vetrano S, Agrillo A, Torroni A, Fabiani F, Iannetti G.Surgical treatment of TMJ ankylosis: our experience (60 cases). J Craniofac Surg. 2002 Jan;13(1): 59-67

3. Gaur A, Singh G, Mishra M, Srivatsan KS and Sachdev V. Distraction osteogenesis for management of obstructive sleep apnea secondary to TMJ ankylosis.. Natl J Maxillofac Surg. 2013; 4(1): 104-106.

4. Okeson JP, editor. Orofacial pain: Guidelines for assessment, diagnosis and management. Chicago: Quintessence; 1996. American Academy of Orofacial Pain; pp. 117pp. 137pp. 141-58

5. Manganello-Souza LC, Mariani PB. Temporomandibular joint ankylosis: Report of 14 cases. Int J Oral Maxillofac Surg. 2003;32(1):24-9.

6. Pertes RA, Gross SG. Disorders of the temporomandibular joint. In: Pertes RA, Gross SG, editors. Clinical management of temporomandibular disorders and orofacial pain. Chicago: Quintessence; 1995. pp. 85-6

7. Topazian RG. Aetiology of temporomandibular joint ankylosis: Analysis of 44 cases. J Oral Surg. 1964;22 : 227-33.

8. Baykul T, Aydin MA, Nasir SN, and Toptas O. Surgical treatment of posttraumatic ankylosis of the TMJ with different pathogenic mechanisms. Eur J Dent. 2012 July; 6(3): 318-323.

9. Abramowicz S, Dolwick MF, Lewis SB, Dolce C. Temporo-mandibular joint reconstruction after failed teflonproplast implant: case report and literature review. Int J Oral Maxillofac Surg. 2008;37(8):763-767.

10. Kearns GJ, Perrott DH, Kaban LB. A protocol for the management of failed alloplastic temporomandibular joint disc implants. J Oral Maxillofac Surg. 1995;53(11):12401247. 
11. Abul-Hassan HS, von Drasek Ascher G, Acland RD. Surgical anatomy and blood supply of the fascial layers of the temporal region. Plast Reconstr Surg. 1986;77(1):17-28.

12. Ahmad QG, Siddiqui RA, Khan AH, Sharma SC. Interposition arthroplasty in temporomandibular joint ankylosis. Indian J Otolaryngol Head Neck Surg. 2004;56 (1):5-8.
13. Felstead AM, Revington PJ, "Surgical Management of Temporomandibular Joint Ankylosis in Ankylosing Spondylitis," International Journal of Rheumatology, vol. 2011, Article ID 854167, 5 pages, 2011

14. Tripathy S, Mohd Yaseen, Singh NN, and Bariar LM. Interposition arthroplasty in post-traumatic temporomandibular joint ankylosis: A retrospective study Indian J Plast Surg. 2009 Jul-Dec; 42(2): 182-187

\section{How to cite this article?}

Bhatnagar S, Navaneetham A. Surgical Management of Tempromandibular Joint Ankylosis: An analysis of 20 cases. Int J Med Res Rev 2014;2(2):110-113. doi:10.17511/ijmrr.2014.i02.07 